\title{
The combination of the loT wireless data acquisition system and edge computation
}

\author{
Ming Yin, and Liwei Wang* \\ Faculty of Electronic and Information, The Southwest Minzu University, Chengdu, 6100041, China
}

Keywords: Internet of things, Edge computation, Data acquisition system, Temperature and humidity sensor

\begin{abstract}
This paper presents an improvement that cominate the algorithm of edge computation and the Internet of things (IoT) data acuisiton system. Firstly, the environment data acquisition system of the agriculture IoT was analyzed and the distributed data processing approach was proposed to handle the abnormal data. we perform the simply processing of the raw acquisition data via the CC2530 by its spare time computation competence. The results prove our algorithm improve the data acquisition efficiency.
\end{abstract}

\section{Introduction}

In recent years, Internet of things related technologies have developed rapidly. At the technical level and application field, the academic and industrial personnel have done a lot of research and development work. The research focuses on the basic definition and characteristics of the Internet of things, the relationship between the Internet of things and sensor networks, ubiquitous networks, the architecture and technical system of the Internet of things [1] [2].

Internet of things technology has been widely used in industrial production, agriculture and animal husbandry, home life and scientific research. Among them, data acquisition system is an important application scene of Internet of things technology. The traditional data acquisition system adopts distributed acquisition technology. Each node collects data separately according to the requirements of the acquisition system, and transmits the data to the central data processing module in the form of data stream. Each data acquisition node works independently, and there is no information exchange and cooperation between them. Single node failure or abnormal data in the data stream can not be processed in the information collection link. After the data is transmitted to the central data processing module, simple processing can be done, which will occupy the limited computing resources of the central data processing module, and delay the subsequent real-time data processing. After the combination of cloud computing and Internet of things technology, the measurement data is transmitted to the cloud module in real time by the Internet of things

* Corresponding author: wangliwei@swun.edu.cn 
system. Some standard data processing algorithms can be carried out by using the powerful computing power of the cloud system. The advantages of cloud computing combined with mobile Internet of things technology can help users build a relatively powerful data acquisition system. The measurement system can be standardized, mobile, and database management system. Cloud system can also establish data visualization and good user interface of the Internet of things.

In the working process of a single data acquisition node, accidental faults and data anomalies caused by power supply or sensor module or external factors are inevitable. Cloud processing these single node failures and exceptions will greatly increase the system overhead and affect the synchronization performance of data streams. The typical terminal data acquisition node is composed of single chip microcomputer or other types of general central processing unit, which often has certain computing power. Under the condition of meeting the working conditions of the Internet of things, using the computation ability of terminal nodes and the principle of edge computation, edge computation can be introduced into the data acquisition system of the Internet of things to optimize the collection system and performance. For example, reduce noise, detect and eliminate abnormal data, and optimize the quality of data stream.

\section{Wireless Data acquisition system of Internet of things with edge computation}

\subsection{Internet of things data acquisition system}

With the development of technology, the traditional data acquisition system has been digitized. Most of them are star topology or hybrid structure. This paper considers a typical agricultural soil data acquisition system, including digital or analog sensor hardware module, terminal control module, digital transmission module, and central data processing module. After the introduction of the Internet of things technology and cloud computing, the Internet of things data acquisition system added cloud server instead of the central data processing module. Wireless sensor network technology is introduced between each node to build a certain scale of small-scale wireless sensor system. The system block diagram is shown in Figure 1.

\subsection{Edge computation}

The concept of edge computing is developed from cloud computing, which refers to the introduction of intermediate edge layer between cloud server and terminal node equipment to deploy computing and storage equipment. The middle edge layer mainly provides local computing, storage and network transmission services [3].

HP lab gives a definition of edge computing in the industry: "edge computing is a scenario in which a large number of heterogeneous wireless (sometimes wireless selforganizing) distributed devices cooperate with each other through the network to complete the computing and storage tasks without the intervention of a third party. These tasks may be related to basic functions such as networks, or to specific applications. Users can rent these distributed devices for a fee to obtain such services "[3].

The main differences between edge computing and cloud computing are compared in many literatures. The main contents of comparison include [4]:

- Distance from the terminal;

- Whether geographical distribution is supported;

- Whether to support data analysis and mining at the edge; 
- Whether to provide high service quality;

- Whether access control is implemented;

- Time delay and power consumption.

\subsection{Data acquisition scheme with edge computation}

Firstly, a data acquisition platform of intelligent agriculture Internet of things is constructed. Hardware structure and software protocol, starting from the bottom, are shown in the table below.

\section{Cloud Server}
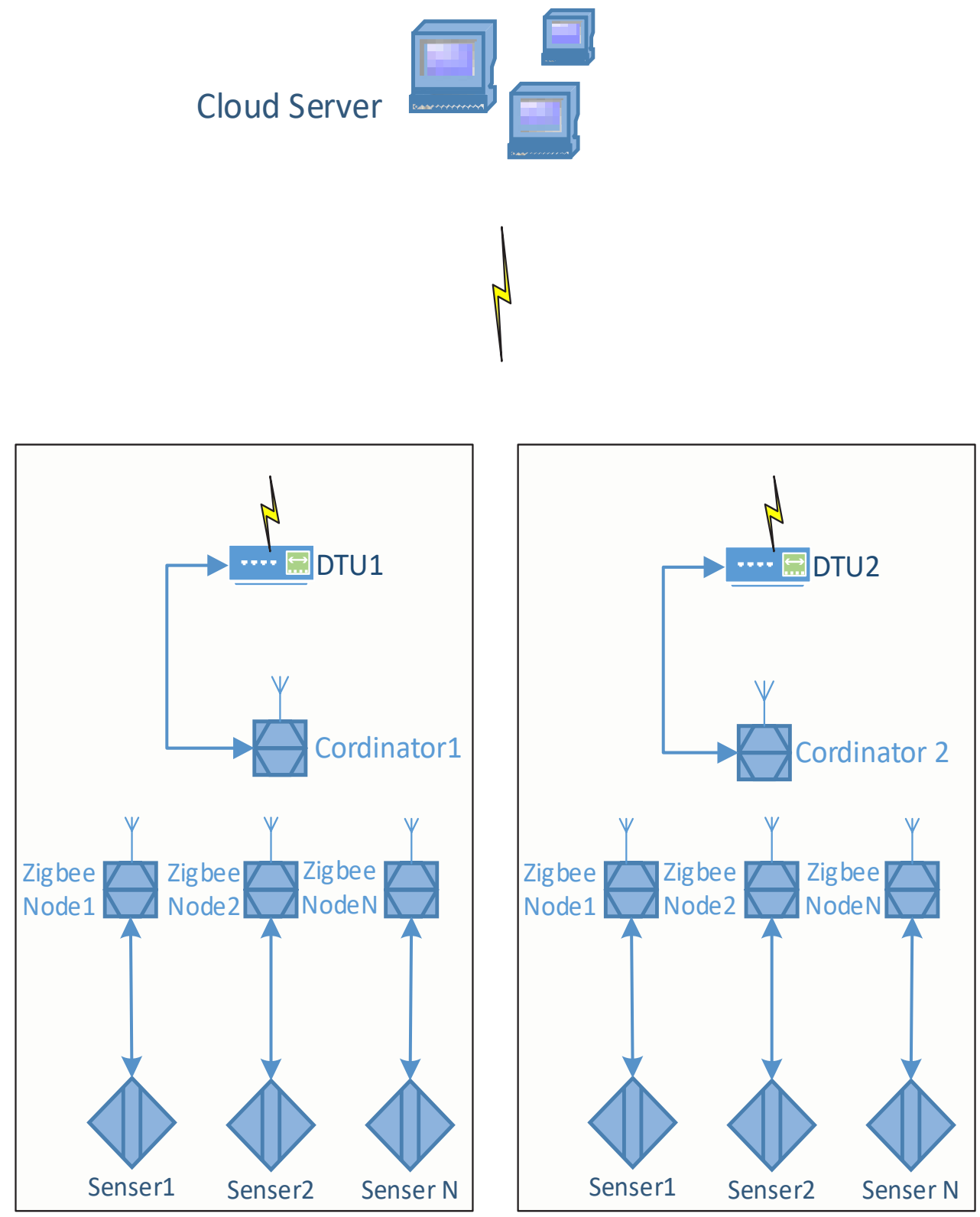

Fig. 1. System view. 
Table 1. Software and hardware composition of data acquisition system.

\begin{tabular}{ll} 
Hardware structure & Software protocol \\
\hline $\begin{array}{l}\text { Digital sensors, Temperature and humidity } \\
\text { sensor }\end{array}$ & $\begin{array}{l}\text { setting working parameters, address } \\
\text { information }\end{array}$ \\
\hline Interface module & set working parameters \\
\hline RS232 or RS485 network & Modbus protocol stack \\
\hline Zigbee chip CC2530 and sub node & ZigBee software protocol stack \\
\cline { 2 - 2 } & edge computation algorithm \\
\hline ZigBee chip CC2530, coordination node & $\begin{array}{l}\text { ZigBee software protocol stack, routing and } \\
\text { interface }\end{array}$ \\
\cline { 2 - 2 } & $\begin{array}{l}\text { edge computation algorithm } \\
\text { data format conversion, transparent wireless } \\
\text { transmission }\end{array}$ \\
Digital transmission module & edge computing, data flow management \\
& Cloud service
\end{tabular}

According to the above table, edge computation is applied to three levels of the whole network structure of the Internet of things, which are ZigBee network sub node, coordination node and wireless digital transmission module of data processing and transmission. Considering the transmission security, the data transmission module is the only transmission relay between cloud module and intermediate node to ensure the transmission transparency. We exclude the digital transmission module from the scope of edge computation, and mainly use ZigBee module as the computation unit.

CC2530 is a powerful system on chip solution for IEEE802.15.4, ZigBee and other applications. With CC2530, powerful network nodes can be built with very low total material cost. CC2530 not only includes excellent performance RF transceiver, core operation unit, enhanced $8051 \mathrm{CPU}$, system programmable flash memory, 8-kbram and many other powerful functions. CC2530 has four different types of flash memory, up to $256 \mathrm{~Kb}$. CC2530 has four different operation modes, which makes it especially suitable for ultra-low power system. In terms of software protocol, ZigBee protocol stack provides a powerful and complete ZigBee solution. At 32m clock, CC2530 provides the computing power of protocol stack and basic control, as well as the computing power for edge computing [5][6][7]8].

In the information data acquisition system, the application examples and algorithms of edge computation are as follows [4].

Data smoothing: A single node obtains data information, mostly strong continuous data. For example, the data of environmental temperature and humidity are abnormal at some time, which obviously leads to data jump. This kind of data point can be monitored and smoothed by the data of before and after time point. Generally, the smoothing value can be obtained by 10 time data points before and after [7].

Data recovery: During the working process of a single node, abnormal data or data loss will occur for a period of time due to sensor failure or electrical failure. In order to balance the data flow, homogeneous data nodes can be used to give data with different weights through ZigBee network to achieve data fitting. So as to recover the time continuous data stream.

Data analysis: Data content monitoring and interesting value extraction are often needed in data acquisition. In cloud computing, it is easy to obtain the above data in data comprehensive analysis. However, cloud computing inevitably encounters time delay and 
problems. For high real-time data monitoring or other special applications, cloud computing can not meet the system requirements. edge computing can avoid this situation. The edge computing unit processes the collected data in real time and cooperates with the cloud server to extract the data of interest or to be monitored.

\section{Conclusions}

This paper introduces the concept and background of edge computing, and applies the edge computing technology to the Agricultural Internet of things data acquisition system. The application example and algorithm analysis of edge computation are given. After verification, the scheme proposed in this paper can effectively help the actual IOT data acquisition system to improve the work efficiency, and the algorithm proposed in this paper can also be applied to other similar IOT data acquisition systems. The power consumption problem of battery power supply in Internet of things nodes and the impact of network congestion caused by network scale expansion on the system are to be solved in the followup work.

This work supported by the Fundamental Research Funds for the Central Universities, Southwest Minzu University, No:2018NQN09.

\section{References}

1. Gubbi, J. , Buyya, R. , Marusic, S. , \& Palaniswami, M. . (2013). Internet of things (iot): a vision, architectural elements, and future directions. Future Generation Computer Systems, 29(7), 1645-1660.

2. Patil, K. A. , \& Kale, N. R. . (2017). A model for smart agriculture using IoT. 2016 International Conference on Global Trends in Signal Processing, Information Computing and Communication (ICGTSPICC). IEEE.

3. You, C. , Huang, K. , Chae, H. , \& Kim, B. H. . (2017). Energy-efficient resource allocation for mobile-edge computation offloading. IEEE Transactions on Wireless Communications, 16(3), 1397-1411.

4. Wei, L., Zhu, H. , Cao, Z. , Dong, X. , Jia, W. , \& Chen, Y. , et al. (2014). Security and privacy for storage and computation in cloud computing. Information ences, 258, 371-386.

5. Ramadan, A. B. , \& Hefnawi, M. . (2018). Adaptation of a data acquisition system for monitoring air quality and radioactivity. The Arab journal of the social ences, 39(1).

6. Guo-Hua, W. , Lian-Zhang, H. , \& Zhi-Cheng, L. . (2018). The system of gateway for collecting wireless data based on cc2530. Electronics World.

7. Ren-Ren, L. I. , Zheng-Hua, Z. , \& Dong-Fang, L. V. . (2017). Design of wireless sensor nodes based on cc2530 and performance analysis. Radio Engineering.

8. Wangqun Jie, Shiyan Ying, Biaobiao Zhang, Jintian Ma, \& Hui Wang. (2012). Development of a zigbee-based wireless sensor network system. IEEE, 727 - 731. 